\title{
Dyeing Properties of Polyurethane-impregnated PET Knit
}

\author{
Jong Ho Park and Sung Dong Kim ${ }^{\dagger}$
}

\author{
Dept of Textile Engineering, Konkuk University, Seoul, 143-701 Korea
}

(Received: September 1, 2008/Revised: September 30, 2008/Accepted: October 20, 2008)

\begin{abstract}
Dyeing and washing fastness properties of polyurethane-impregnated polyester (PU-impregnated PET), and the distribution of two disperse dyes between PET and PU film were studied to investigate the effect of PU portion to exhaustion and washing fastness. Dyeing properties of PU-impregnated PET were quite different with those of PET: PU-impregnated PET absorbed disperse dye linearly from the early stage of dyeing to equilibrium, and it exhibited excellent build-up property up to $4 \%$ owf dyeing: The absorbed dye on PU film at early dyeing stage migrated to more substantive PET at the temperature higher than 115 . The amount of exhausted dye on PET portion was larger than on PU film and the distribution ratio was 2.08 2.34. The grade of washing fastness of PU-impregnated PET was the same as or lower by 0.5 1 grade than PU film whose washing fastness was lower by $0.5 \sim 1$ than PET.
\end{abstract}

Keywords: disperse dye, dyeing property, washfastness, PU, PET

\section{Introduction}

Polyurethane(PU) consists of hard segment and soft segment ${ }^{1)}$. The hard segment has urethane bonds(-NHCOO-) or urea bonds(-NHCONH-). These bonds of each molecular chains form intermolecular hydrogen bonds which contribute to good tensile strength, chemical and thermal resistance. The soft segment is made of repeating aliphatic polyester or aliphatic polyether units which do not have strong intermolecular interaction, resulting in amorphousness which is related to good flexible and stretching properties. Because of its good chemical and physical properties PU has been widely applied in wateproof paint, thermal insulator, coating resin for electronics and coating agent for imitation leather which is very useful to shoes, furniture and clothes $^{2)}$.

Substantial research efforts had been directed towards selecting appropriate dyes and dyeing processes for PU since Bayer announced commercial application of $\mathrm{PU}^{3,4)}$. As a result, it has been known that several types of dye have affinity to PU.
As PU fiber and resin are used in textile industry not by itself but by combining with other fibers, the staining PU with dyes for other fibers resulted in lowering washing fastness. The use of cationic complex agent was examined for dyeing PU-nylon products with acid dyes ${ }^{5}$. Improvement of wet fastness of PU-nylon or PU-polyester products had been pursued by low temperature dyeing utilizing low energy type disperse dye ${ }^{6}$, or by developing new disperse dyes ${ }^{7}$.

When PU is used as resin for imitation leather, PET fabric is impregnated with PU solution and then it is dyed with disperse dye. PU is so hydrophobic that it also has affinity to the hydrophobic disperse dye. The disperse dye is absorbed not only on PET but also on PU during dyeing process. The disperse dyes staining PU have been recognized as the main reason for low washing fastness of dyed imitation leather products. Although PU has been used in textile industry for a long time, few studies were carried out on the distribution of dye or on the effect of PU portion to the color fastness of PU-polyester or PU-nylon products. 
Only recently, Qian et al has studied the distributions between PU fiber and polyester, and polyamide fibers using a range of monoazo disperse dyes $^{7}$. Disperse dyes showed different dyeing properties with their chemical structure ${ }^{8,9)}$. It is critical to select the right dye for successful dyeing.

The purpose of the present study is to investigate the dyeing and fastness properties of PU-i mpregnated PET knit with two disperse dyes. The distribution of disperse dyes between PET and PU, and effect of absorbed dyes on PU to deteriorate washing fastness of PU-impregnated PET have also been examined.

\section{Experimental}

\subsection{Materials}

PET circular knit (sea-island type 65d/204f), PU film which was made from PU resin (GT-300LV, PTMG-based type), PU-impregnated PET knit (weight ratio of PET : PU $=80: 20$ ) were used for dyeing. Two disperse dyes such as Foron Yellow Brown S-2RFL (C. I. Disperse Orange 30) and Foron Rubine S-2GFL (C. I. Disperse Red 167) were used. Their chemical structures are shown in Fig. 1. Sandogen LDK(Clariants) was used as a dispersing agent.

Acetic acid, sodium acetate, sodium hydrosulphite and sodium hydroxide used were first grade. Organic solvent like dimethylformamide(DMF) and acetone which were used for extraction of disperse dye were commercial grade.

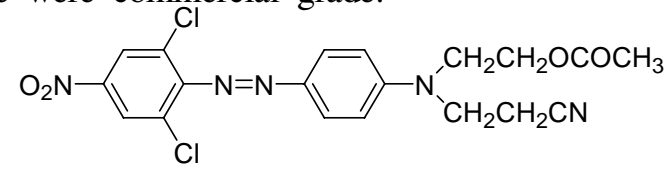

Foron Yellow Brown S-2RFL

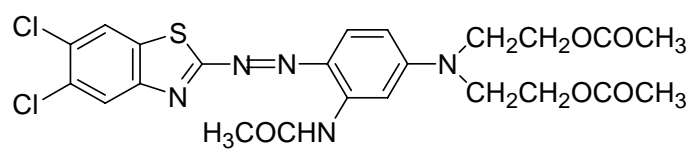

Foron Rubine S-2GFL

Fig. 1. Chemical structure of disperse dyes used in this study.

| 36 | www.ksdf.or.kr

\subsection{Fabrication of PU film}

PU resin was diluted with DMF by 50\% and casted on glass plate with knife. And it was dried in a vacuum oven at ${ }^{\circ} \& 0$ for 24 hours.

\subsection{Dyeing}

Dye bath $\mathrm{pH}$ was adjusted to $4 \sim 4.5$ by employing acetic acid/sodium acetate buffer system. Dyeing was carried out in the sealed, stainless steel dye pots with $120 \mathrm{~cm}^{3}$ capacity of Starlet II (DL-6000, Daelim). Samples were placed at 25 in a dye bath of liquor ratio $20: 1_{\mathrm{C}}$ Cemperature was raised to 13 0 by the rate of 1.5 per minute, and the dyeing was continued for $60 \mathrm{~min}$. Dyed fabric was reduction cleared in an aqueous solution containing $2 \mathrm{~g} / \mathrm{L}$ sodium hydroxide and $2 \mathrm{~g} / \mathrm{L}_{\mathrm{C}}$ sodium hydrosulphite using a liquor ratio $1: 40$ at 80 for $20 \mathrm{~min}$.

The rates of dyeing were determined at $1 \%$ owf depth with Dye-O-Meter (DyeMax-L, Dyetex Engineering). DyeMax-L is a real-time dyeing measuring system which can monitor the extent of exhaustion at predetermined time intervals by assessing the residual dye bath absorbance. The build-up property was examined by comparing $\mathrm{K} / \mathrm{S}$ values of the samples dyed at the concentration of $0.5,1,2$ and 4 \%owf. $0.8 \mathrm{~g}$ of PET and $0.2 \mathrm{~g}$ of PU film was dyed at $1 \%$ owf in the same bath to determine distribution of dye between two silbstrates.

Heat setting was done at 130 for 150 seconds without tension.

\subsection{Measurement of dye uptake and washing fastness}

$\mathrm{K} / \mathrm{S}$ values of the dyed samples were calculated from the reflectance curves measured using a spectrophotometer(Color-Eye 3100, Macbeth) interfaced with a personal computer. The settings were as follows: specular light included, large area of view, UV included, standard light D65 and $10^{\circ}$ standard observer view.

To calculate the amount of disperse dye in dyed samples, DMF and acetone were used as extractant for PET and PU film and the absorption of dye solution was measured using UV/Vis spectro- 
photometer (Shimadzu).

Fastness to washing was measured using ISO 105-C06/A2S method.

\section{Results and discussion}

\subsection{Dyeing properties of PU-impregnated PET}

$\mathrm{K} / \mathrm{S}$ values of PU-impregnated PET knit dyed at $0.5,1,2,4 \%$ owf were measured to investigate the build-up property of disperse dyes. Fig. 2 shows $\mathrm{K} / \mathrm{S}$ values of the samples dyed with two disperse dyes. $\mathrm{K} / \mathrm{S}$ value of two dyes increases almost in proportional to the dye concentration, which indicates their buil-up properties are very good. It is usually observed in PET dyeing that increase in K/S value slows down above dye concentration higher than 2 \%owf. The excellent dye uptake might be caused by PU portion in samples.

The extents of exhaustion as a function of dyeing time were measured on Dye-O-Meter to examine the rate of dyeing of PU-impregnated PET in comparison with that of PET. Fig. 3 demonstrates exhaustion curves of two disperse dyes on PET and PU-impregnated PET. The amount of exhausted disperse dyes on PET increased (S)lowly at low temperature but after $30 \min (70$ ) increased dramiatically and reached equilibrium after $60 \mathrm{~min}$ (115 ). The exhaustion curves of PU-impregnated PET differed from those of PET. The amount of exhausted dye increased linearly from early stage of dyeing to equilibrium. The time required to reach equilibrium was $10 \mathrm{~min}$ shorter than PET. The difference in dye exhaustion profile might be caused by PU component. The ${ }^{\circ} \mathrm{PTMG}-$ based PU fibers have a Tg ranging from $-60{ }^{\circ} \mathrm{C}$ to -30 which is way below that of PEF $(70 \sim 80$ ). The dyeing tempera ture at the $\operatorname{start}(25)$ is so higher than $\mathrm{Tg}$ of $\mathrm{PU}$ that disperse dye penetrated into the amorphous region of PU component without difficulty. Hence the PU component in PU-impregnated PET easily absorbed disperse dye even in early stage of dyeing.

The molecular weight of Foron Yellow Brown and Foron Rubine are $450 \mathrm{~g} / \mathrm{mol}$ and $506 \mathrm{~g} / \mathrm{mol}$ respectively. The dyeing rate of Foron Yellow Brown was faster than that of Foron Rubine, but the final extent of exhaustion was lower. This might be caused by the fact that Foron Rubine is bulkier than Foron Yellow Brown as shown in Fig. 1.

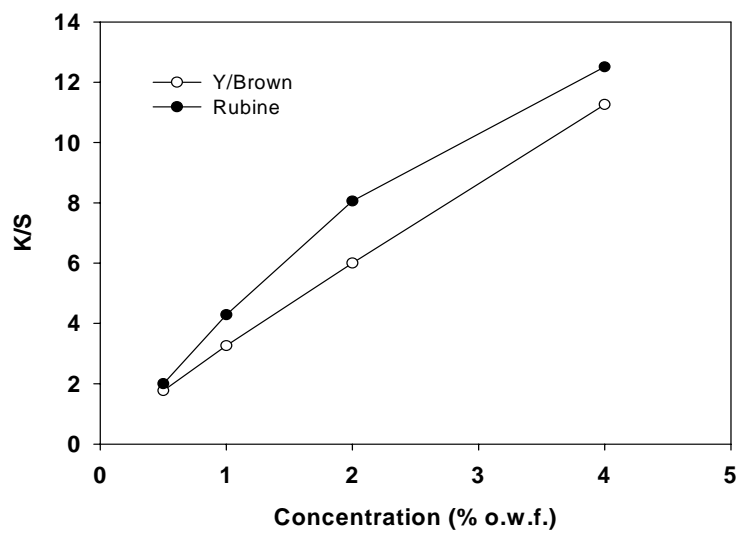

Fig. 2. Build-up properties of PU-impregnated PET knit.

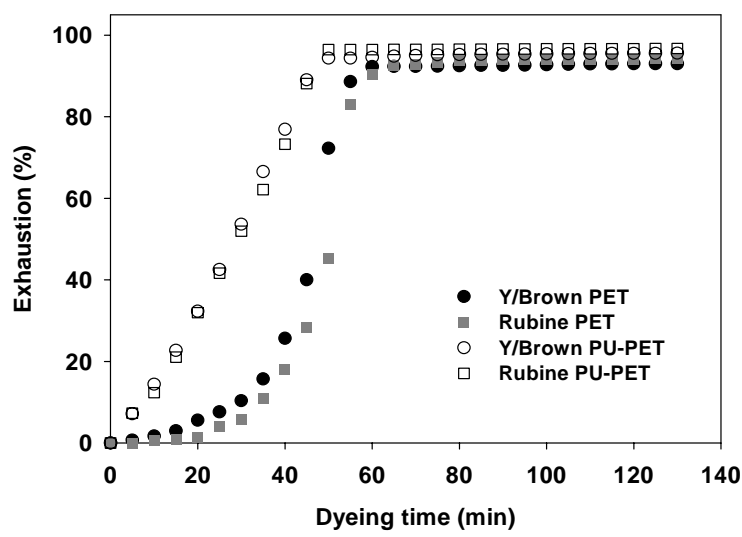

Fig. 3. Exhaustion curves of PET and PU-impregnated PET.

\subsection{Distribution of disperse dye between PU film and PET in the same bath dyeing}

When PU-impregnated PET was dyed it was impossible to determine the distribution of disperse dye between PET and PU. Therefore PET and PU film (weight ratio was 80:20) were dyed at the same dyebath and the amounts of exhausted dye on each substrate were measured by extracting with organic solvents. The amount of exhausted dye on PET, PU film dyed with Foron Yellow Brown and sum of them are shown in Fig. 4. The extent of exhaustion of the dye on PU film increased ${ }^{\circ} \mathrm{C}$ rapidly from the beginning of dyeing to $60 \min (115)$ and then decreased sharply. The extent of the dye on PET increased slowly at the early stage of dyeing and $J$. of the Korean Soc. of Dyers and Finishers, Vol. 20, No. 5 | 37 | 


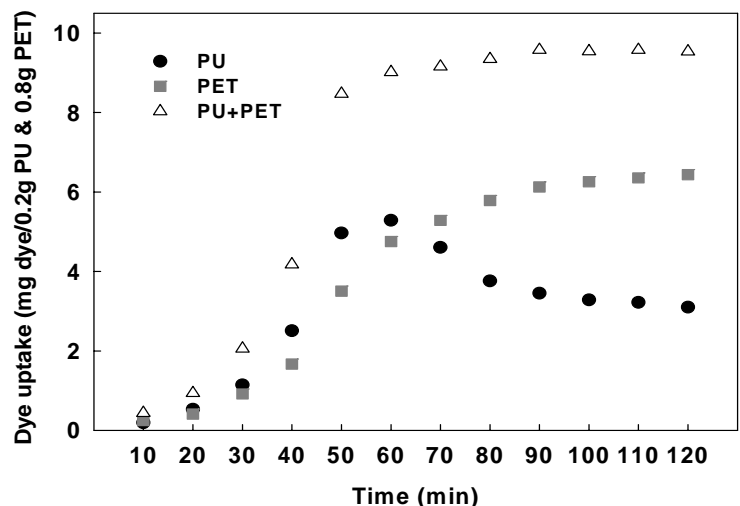

Fig. 4. Distribution of Foron Y/Brown S-2RFL between PET and PU film.

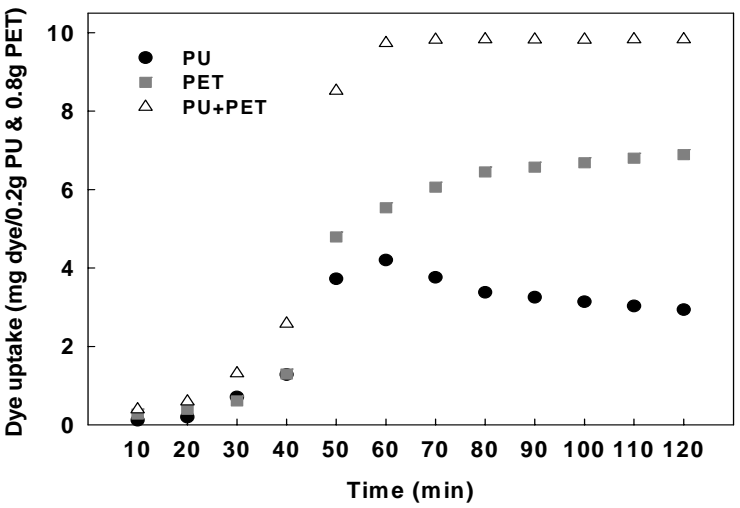

Fig. 5. Distribution of Foron Rubine S-2GFL between PET and PU film.

increasedC rapidly in the 'emperature range from $30 \mathrm{~min}\left(70\right.$ ) to ${ }^{\circ} 60 \mathrm{~min}(115)$, and continued to increase over 115 . The experimental result suggests that the disperse dye which was absorbed on PU film at early dyeikig stage migrated to more substantive PET over 115 . Considering the weight ratio of PU, the amount of exhausted dye on unit weight of PU film was higher than unit weight of PET.
The exhaustion curves of Foron Rubine(Fig. 5) showed almost the same tendency to those of Foron Yellow Brown. The careful examination revealed that the amount of exhausted Foron Rubine on PU film until $11^{\circ} \quad(4.20 \mathrm{mg})$ was $1.09 \mathrm{mg}$ less than that of Foron Yellow brown $(5.29 \mathrm{mg})$, and that the amount of exhausted Foron Rubine on PET until ${ }_{115} \mathrm{C}$ was higher than that of Foron Yellow Brown. Considering that molecular weight of Foron Rubine was higher than Foron Yellow Brown, it could be said that the amont of exhausted dye on PU become less, but the amount of exhausted on PET become more due to the increased interaction with PET. The disperse dyes having different molecular size were prepared and their dyeing properties on PUimpregnated PET showed the same tendency ${ }^{10}$.

After the dyeing process was completed, it was found that the amount of exhausted Yellow Brown on $0.8 \mathrm{~g}$ of PET and $0.2 \mathrm{~g}$ of PU film were $6.44 \mathrm{mg}$ and $3.10 \mathrm{mg}$ respectively, and the dye distribution ratio was 2.08. The amount of exhausted Foron Rubine on $0.8 \mathrm{~g}$ of PET and $0.2 \mathrm{~g}$ of PU film were $6.89 \mathrm{mg}$ and $2.94 \mathrm{mg}$ respectively, and the dye distribution ratio was 2.34 .

\subsection{Influence of PU component of PU- impregnated PET upon washing fastness}

The grades of washing fastness of PU film, PET and PU-impregnated PET are listed in Table 1. Although multi-fabrics are used for washing fastness test, the grades evaluated by the staining on nylon which is easily contaminated by disperse dyes are discussed here.

Table 1. Dye amounts in substrates after dyeing, after $\mathrm{R} / \mathrm{C}$ and after washing

\begin{tabular}{|c|c|c|c|c|c|c|}
\hline & \multicolumn{3}{|c|}{ Yellow Brown S-2RFL } & \multicolumn{3}{|c|}{ Rubine S-2GFL } \\
\hline Amount of dye (mg) & After dyeing & After $\mathrm{R} / \mathrm{C}$ & After wash & After dyeing & After $\mathrm{R} / \mathrm{C}$ & After wash \\
\hline in PET & 6.68 & 6.53 & 6.42 & 7.02 & 6.89 & 6.80 \\
\hline lost during washing & & 0.15 & 0.11 & & 0.13 & 0.09 \\
\hline lost during washing(\%) & & 2.25 & 1.68 & & 1.85 & 1.31 \\
\hline in $\mathrm{PU}$ & 2.74 & 2.56 & 2.20 & 2.58 & 2.41 & 2.10 \\
\hline lost during washing & & 0.18 & 0.36 & & 0.16 & 0.31 \\
\hline lost during washing(\%) & & 5.11 & 14.06 & & 6.20 & 12.86 \\
\hline in both substrate & 9.42 & 9.09 & 8.62 & 9.60 & 9.30 & 8.90 \\
\hline tost during washimg & & 0.33 & 0.47 & & 0.30 & 0.40 \\
\hline lost during washing $(\%)$ & & 3.50 & 5.17 & & 3.13 & 4.30 \\
\hline
\end{tabular}

| 38 | www.ksdf.or.kr 
Washing fastness of substrates dyed with Foron Yellow Brown and Foron Rubine showed the same tendency that the washing fastness grade of PET the best, and that of PU was lower by $0.5 \sim 1$ grade, and that of PU-impregnated PET was lower than PU film by $0.5 \sim 1$ grade than PU film. These experimental results indicated that washing fastness of PU-impregnated PET wass influenced by PU component. The grades of washing fastness of Foron Yellow Brown were better than those of Foron Rubine.

Fig. 6 and Table 2 show the amount of dye in substrates before and after reduction clearing of PET and PU film (weight ratio 80:20) dyed in the same bath, and sum of them. The amount of Foron Yellow Brown removed from PET after reduction clearing was $0.15 \mathrm{mg}(2.25 \%)$ and that after washing fastness test was $0.11 \mathrm{mg}(1.68 \%)$. The amount of Foron Rubine removed from PET after reduction clearing was $0.13 \mathrm{mg}(1.85 \%)$ and that after washing fastness test was $0.09 \mathrm{mg}(1.31 \%)$. In the case of PET the amounts of dye removed by washing were less than those by reduction clearing. But the amount of Foron Yellow Brown removed from PU film after

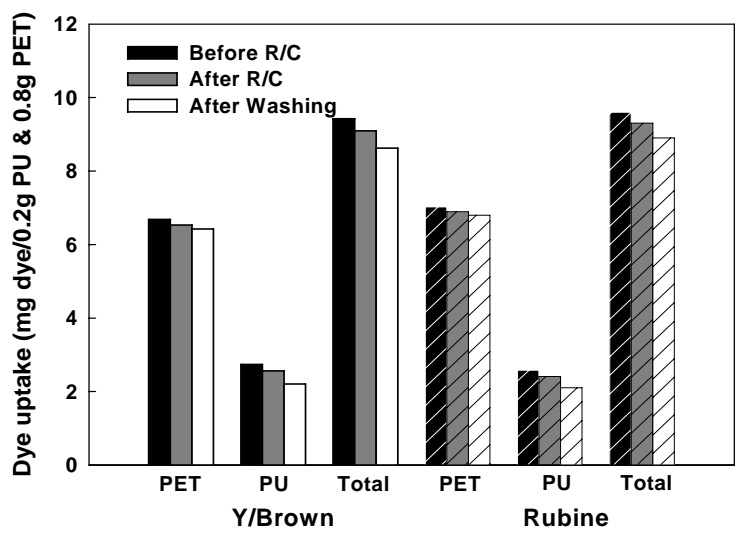

Fig. 6. Dye amounts in substrates before R/C, after $\mathrm{R} / \mathrm{C}$ and after washing. reduction clearing was $0.18 \mathrm{mg}(5.11 \%)$ and that after washing fastness test was $0.36 \mathrm{mg}(14.06 \%)$. The amount of Foron Rubine removed from PU film after reduction clearing was $0.16 \mathrm{mg}(6.20 \%)$ and that after washing fastness test was $0.31 \mathrm{mg}(12.86 \%)$. In the case of PU the amount of dye removed by washing was much more than that by reduction clearing, suggesting that desorption of dye from PU component migt be the main reason for the lower grade of PU film and PU-impregnated PET. These results also mean that most of dyes adhering on the surface of PET can be eliminated by reduction clearing, but one third of the dyes contaminating PU is eliminated by reduction clearing and two thirds of the dyes contaminating PU are removed by washing.

\section{Conclusion}

Dyeing and fastness properties of the PU-impregnated PET were studied with two disperse dyes such as Foron Yellow Brown S-2RFL and Foron Rubine S-2GFL to elucidate the reason for low washing fastness, and concluded as follows:

1. The exhaustion curves of PU-impregnated PET differed from those of PET: The amount of exhausted dye increased linearly from early stage of dyeing to equilibrium, and PU-impregnated PET exhibited excellent build-up property up to 4 \%owf dyeing.

2. It was found that he absorbed dye on PU film at early dyeing stage migrated to more substantive PET at high temperature in the one bath dyeing process, and that the amount of exhausted dye on PET portion was twice as much as the amount of exhausted dye on PET portion.

3. The grade of washing fastness of PU-impregnated PET was the same as or lower by $0.5 \sim 1$ grade than PU film whose washing fastness was lower by $0.5 \sim 1$ than PET.

Table 2. Grades of washing fastness of PET, PU and PU-impregnated PET

Yellow Brown S-2RFL

Rubine S-2GFL

\begin{tabular}{|c|c|c|c|c|c|c|c|c|c|c|c|c|}
\hline & wool & acryl & PET & nylon & cotton & acetate & wool & acryl & PET & nylon & cotton & acetate \\
\hline PET & $4 \sim 5$ & 5 & $4 \sim 5$ & $4 \sim 5$ & $4 \sim 5$ & $4 \sim 5$ & $4 \sim 5$ & $4 \sim 5$ & $3 \sim 4$ & 3 & $3 \sim 4$ & $3 \sim 4$ \\
\hline PU & 4 & 4 & $3 \sim 4$ & $3 \sim 4$ & 4 & 3 & 3 & 3 & $2 \sim 3$ & $2 \sim 3$ & 3 & $2 \sim 3$ \\
\hline PU-impregnated PET & 4 & 4 & 3 & $2 \sim 3$ & $3 \sim 4$ & 3 & 3 & 3 & $2 \sim 3$ & 2 & 3 & $2 \sim 3$ \\
\hline
\end{tabular}




\section{References}

1. H. Oertel, Elastomeric Threads containing Polyurethanes: Their Structure, Properties, and Uses, Melliland Textilber., 46, 51-59(1965).

2. D. Y. Kim, "Polyurethanes: PU Science \& Technology", The Polyurethane Society of Korea, pp.399-412, 2006.

3. H. L. Hanna, Dyeing of Polyurethane Elastomeric Materials in Nonaqueous Media, Textile research journal, 45, 573-576(1975).

4. R. W. Monclieff, "Man-Made Fiber", NewnesButterworths, 6th ed., pp.493-494, 1975.

5. J. Shore, "Blends Dyeing", S.D.C., Bradford, p.35, 1998.

6. J. H. Choi, A. D. Towns, Acetate Dyes Revisited: High Fastness Dyeing of Cellulose Diacetate and Polyester-Polyurethane, Coloration Technology, 117(3), 127-133(2001).
7. H. F Qian, X. Y. Song, The Structure of Azo Disperse Dyes and its Distribution of Polyurethane Fiber Blend with Polyester, or Polyamide Fiber, Dyes and Pigments, 74, 672-676 (2007)

8. S. D. Kim, M. J. Kim and Y. S. Lee, Synthesis and Application of New Disperse Dyes for Micropolyester Fabric, Journal of the Korean Fiber Society, 37, 180-189(2000).

9. S. D. Kim, M. J. Kim, B. S. Lee, and K. S. Lee, Effects of Thermomigration on the Washfastness of Disperse Dyes Having Different Molecular Size, Fibers and Polymers, 5(1), 39-43(2004).

10. J. H. Park and S. D. Kim, Dyeing Properties of Polyurethane-coated Polyester with Disperse Dyes, Textile Science and Engineering, 45(3), 186-191(2008). 